Y. Teranishi

Nagoya Math. J.

Vol. 95 (1984), 137-161

\title{
THE VARIATIONAL THEORY OF HIGHER-ORDER LINEAR DIFFERENTIAL EQUATIONS
}

\author{
YASUO TERANISHI
}

\section{$\S 1$. Introduction}

In his paper [2], [3], D. A. Hejhal investigated the variational theory of linear polynomic functions. In this paper we are concerned with the variational theory of higher-order differential equations. To be more precise, consider a compact Riemann surface having genus $g>1$. As is well known, we can choose a projective coordinate covering $\mathfrak{A}=\left(U_{\alpha}, z_{\alpha}\right)$. Fix this coordinate covering of $X$. We shall be concerned with linear ordinary differential operators of order $n$ defined in each projective coordinate open set $U_{\alpha}$

$$
L_{n, \alpha}\left(P_{\alpha} \mid z_{\alpha}\right)=\left(\frac{d}{d z_{\alpha}}\right)^{n}+\sum_{\ell=1}^{n}\left(\begin{array}{c}
n \\
\ell
\end{array}\right) P_{n, \alpha}\left(z_{\alpha}\right)\left(\frac{d}{d z_{\alpha}}\right)^{n-\ell}
$$

where coefficients $P_{1, \alpha}\left(z_{\alpha}\right), \cdots, p_{n, \alpha}\left(z_{\alpha}\right)$ are holomorphic in $U_{\alpha}$. Differential operators $\left\{L_{n, \alpha}\left(P_{\alpha} \mid z_{\alpha}\right)\right\}$ are called a semi-canonical form if $P_{1, \alpha}\left(z_{\alpha}\right)=0$ for all $\alpha$.

Let $\lambda \in H^{1}\left(X, \mathcal{O}_{x}\right)$ be a complex line bundle on $X$. Differential operators $\left\{L_{n, \alpha}\left(P_{\alpha} \mid z_{\alpha}\right)\right\}$ are called $\lambda$-related if in each intersection $U_{\alpha} \cap U_{\beta}$

$$
L_{n, \alpha}\left(P_{\alpha} \mid z_{\alpha}\right) y=\left(\frac{d z_{\beta}}{d z_{\alpha}}\right)^{n} \lambda_{\alpha \beta}(z)^{-1} L_{n, \beta}\left(P_{\beta} \mid z_{\beta}\right) \lambda_{\alpha \beta}\left(z_{\beta}\right) y .
$$

We shall prove an analogous theorem of the Laguerre-Forsyth's basic differential invariants.

TheOREM 1.1. Let $\left\{L_{n, \alpha}\left(P_{\alpha} \mid z_{\alpha}\right)\right\}$ be a $\lambda$-related semi-canonical form, then

$$
\left(\theta_{m, \alpha}\left(z_{\alpha}\right)\right) \in \Gamma\left(\mathfrak{A}, \mathcal{O}\left(\kappa^{m}\right)\right) \quad(m=2,3, \cdots, n)
$$

where $\theta_{m, \alpha}\left(z_{\alpha}\right)$ is holomorphic function in $U_{\alpha}$ defined by:

$$
\theta_{m, \alpha}\left(z_{\alpha}\right)=\frac{1}{2} \sum_{k=0}^{m-2}(-1)^{k} \frac{(m-2) ! m !(2 m-k-2) !}{(m-k-1) !(m-k) !(2 m-3) ! k ! !}\left(\frac{d}{d z_{\alpha}}\right)^{k} P_{m-k, \alpha}\left(z_{\alpha}\right) .
$$

Received August 19, 1983. 
Conversely for given $\theta_{m}(z) \in \Gamma\left(\mathfrak{H}, \mathcal{O}\left(\kappa^{m}\right)\right)(m=2,3, \cdots, n)$, we can define differential polynomials of $\left\{\theta_{m}(z)\right\}$

$$
P_{2, \alpha}\left(z_{\alpha}\right), \quad P_{3, \alpha}\left(z_{\alpha}\right), \cdots, P_{n, \alpha}\left(z_{\alpha}\right) .
$$

And differential operators $\left\{L_{n, \alpha}\left(P_{\alpha} \mid z_{\alpha}\right)\right\}$ are a $\lambda$-related semi-canonical form.

If $\varphi_{1, \alpha}\left(z_{\alpha}\right), \cdots, \varphi_{n, \alpha}\left(z_{\alpha}\right)$ are $n$ linearly independent holomorphic solutions of the $\lambda$-related semi-canonical differential equation $L_{n, \alpha}\left(P_{\alpha} \mid z_{\alpha}\right) y=0$ and $\sigma$ is any element of $S L(n, C)$ then the vector valued holomorphic function $\varphi_{\alpha}\left(z_{\alpha}\right)={ }^{t}\left(\varphi_{1, \alpha}\left(z_{\alpha}\right), \cdots, \varphi_{n, \alpha}\left(z_{\alpha}\right)\right)$ satisfies in each intersection $U_{\alpha} \cap U_{\beta}$

$$
\varphi_{\alpha}\left(z_{\alpha}\right)=\rho_{\alpha \beta}\left(c_{\alpha \beta} z_{\beta}+d_{\alpha \beta}\right)^{1-n} \varphi_{\beta}\left(z_{\beta}\right)
$$

for some $\rho_{\alpha \beta} \in S L(n, C)$, and the composition $\varphi_{\alpha}^{\prime}=\sigma \circ \varphi_{\alpha}$ satisfies a relation of the form (1.1) with $\rho_{\alpha \beta}$ replaced by $\rho_{\alpha \beta}^{\prime}=\sigma \circ \rho_{\alpha \beta} \circ \sigma^{-1}$. Therefore the mapping $\varphi_{\alpha}$ and $\varphi_{\alpha}^{\prime}$ are considered as describing equivalent flat vector bundles of rank $n$ on $X$.

There is a natural one to one correspondence between the cohomology set $H^{1}(X, S L(n, C))$ and the quotient space $\operatorname{Hom}\left(\pi_{1}(X), S L(n, C) / S L(n, C)\right.$, where $S L(n, C)$ acts on Hom $\left(\pi_{1}(X), S L(n, C)\right)$ by inner automorphisms. The homomorphism obtained from a $\lambda$-related semi-canonical equation is called a monodromy representation. The fundamental group $\pi_{1}(X)$ is described canonically as a group with $2 g$ generators $\sigma_{1}, \cdots, \sigma_{g}, \tau_{1}, \cdots, \tau_{g}$ and the commutator relation $\left[\sigma_{1}, \tau_{1}\right] \cdots\left[\sigma_{g}, \tau_{g}\right]=1$.

Introducing the complex variety

$$
N=\left\{\left(X_{1}, \cdots, X_{g}, Y_{1}, \cdots, Y_{g}\right) \in S L(n, C)^{2 g} \mid\left[X_{1}, Y_{1}\right] \cdots\left[X_{g}, Y_{g}\right]=1\right\},
$$

the mapping which associates to an element $\rho \in \operatorname{Hom}\left(\pi_{1}(X), S L(n, C)\right)$ the point $\left(\rho\left(\sigma_{1}\right), \cdots, \rho\left(\sigma_{g}\right), \rho\left(\tau_{1}\right), \cdots, \rho\left(\tau_{g}\right)\right) \in N$ identifies $\operatorname{Hom}\left(\pi_{1}(X), S L(n, C)\right)$ with the complex analytic variety $N$ and the cohomology set $H^{1}(X, S L(n, C))$ with the quotient space $V=N / S L(n, C)$ where $S L(n, C)$ acts on $N$ by inner automorphisms. (See Gunning [5]). The tangent space to the variety $V$ at a point corresponding to the monodromy representation $\rho$ of a $\lambda$ related semi-canonical differential equation is identified with the cohomology group $H^{1}\left(\pi_{1}(X), \operatorname{Ad}_{0} \rho\right)$ of the group $\pi_{1}(X)$ with coefficients in the space of $n \times n$ matrices of trace zero under the group representation Ad $\rho$.

By Theorem 1.1, we can introduce a complex analytic mapping between the vector space $\oplus_{m=2}^{n} \Gamma\left(X, \mathcal{O}\left(\kappa^{m}\right)\right)$ and the variety $N$, and the tangent 
space to the image at a monodromy representation $\rho \in N$ is identified with the space of the period classes of the Prym differentials $\Gamma\left(X, \mathcal{O}^{1,0}\left(\operatorname{Ad}_{0} \rho\right)\right)$. We shall introduce the transvectants which are known in the classical invariant theory and using them describe the period classes of the Prym differentials (The variational formula).

The formulas also suggest a close relationship with Eichler cohomology groups. Many formulas in this paper can be found in Hejhal ([2], [3]) under the restriction $2 \leqslant n \leqslant 6$ and we shall eliminate this restriction using invariant theoretic method.

The author wishes to express his hearty thanks to Professors H. Morikawa and H. Popp for their kind advices and encouragements.

\section{§2. The basic differential equations and the monodromy represen- tation}

Let $X$ be a compact Riemann surface of genus $g>1$. Since the genus of $X$ is greater than one, as is well known we can find a coordinate covering $\mathfrak{X}=\left\{\left(U_{\alpha}, z_{\alpha}\right)\right\}$ such that the coordinate transformations of this coordinate covering are projective linear transformations

$$
z_{\alpha}=\frac{a_{\alpha \beta} z_{\beta}+b_{\alpha \beta}}{c_{\alpha \beta} z_{\beta}+d_{\alpha \beta}} \quad\left(\begin{array}{ll}
a_{\alpha \beta} & b_{\alpha \beta} \\
c_{\alpha \beta} & d_{\alpha \beta}
\end{array}\right) \in S L(2, C) \text { in } U_{\alpha} \cap U_{\beta} .
$$

Such a coordinate covering $\mathfrak{A}$ is called a projective coordinate covering of $X$.

We denote by $L_{\alpha}(n)$ the set of all the homogeneous monic linear differential operators of degree $n$ defined in a projective coordinate open set $U_{\alpha}$ :

$$
L_{n, \alpha}\left(P_{\alpha} \mid z_{\alpha}\right)=\left(\frac{d}{d z_{\alpha}}\right)^{n}+\sum_{\ell=1}^{n}\left(\begin{array}{c}
n \\
\ell
\end{array}\right) P_{\ell, \alpha}\left(z_{\alpha}\right)\left(\frac{d}{d z_{\alpha}}\right)^{n-\ell}
$$

where coefficients $P_{1, \alpha}\left(z_{\alpha}\right), \cdots, P_{n, \alpha}\left(z_{\alpha}\right)$ are holomorphic on $U_{\alpha}$ and $\left(\begin{array}{c}n \\ \ell\end{array}\right)$ is the binomial coefficient.

We denote by $L_{n}(P \mid z)$ the collection of local differential operators:

$$
L_{n}(P \mid z)=\left(L_{n, \alpha}\left(P_{\alpha} \mid z_{\alpha}\right)\right),
$$

and denote by $\mathscr{L}(n)$ the set of all $L_{n}(P \mid z)$.

For a given complex line bundle $\lambda \in H^{1}\left(X, \mathcal{O}^{\times}\right)$, we associate an element $L_{n}(P \mid z) \in L(n)$ which satisfies the following transformation relation in $U_{\alpha}$ $\cap U_{\beta}$ 


$$
L_{n, \alpha}\left(P_{\alpha} \mid z_{\alpha}\right) y=\left(\frac{d z_{\beta}}{d z_{\alpha}}\right)^{n} \lambda_{\alpha \beta}\left(z_{\beta}\right)^{-1} L_{n, \beta}\left(P_{\beta} \mid z_{\beta}\right) \lambda_{\alpha \beta}\left(z_{\beta}\right) y
$$

Definition 2.1. Differential operators $L_{n}(P \mid z)$ satisfying the relation (2.4) is called a $\lambda$-related differential operator.

Definition 2.2. Differential operators $L_{n}(P \mid z) \in L(n)$ is called a semicanonical form if $P_{1, \alpha}\left(z_{\alpha}\right)=0$ for all $\alpha$.

LEMma 2.1. Let $\lambda \in H^{1}\left(X, \mathcal{O}^{\times}\right)$be a complex line bundle on $X$, and $L_{n}(P \mid z)$ be a $\lambda$-related differential operator. Then

$$
\begin{aligned}
P_{1, \alpha}\left(z_{\alpha}\right)=\left(\frac{d z_{\alpha}}{d z_{\beta}}\right)^{-1}\left\{P_{1, \beta}\left(z_{\beta}\right)\right. & +\lambda_{\alpha \beta}^{-1}\left(z_{\beta}\right) \frac{d}{d z_{\beta}} \lambda_{\alpha \beta}\left(z_{\beta}\right) \\
& \left.+\frac{n-1}{2}\left(\frac{d z_{\alpha}}{d z_{\beta}}\right)^{-1}\left(\frac{d}{d z_{\beta}}\right)^{2} z_{\alpha}\right\} .
\end{aligned}
$$

For the proof, see ([1], Lemma 3.5).

Proposition 2.1. Let $\lambda \in H^{1}\left(X, \mathcal{O}^{\times}\right)$be a complex line bundle on $X$, and $L_{n}(P \mid z)$ be a $\lambda$-related semi-canonical form. Then

$$
\text { degree }(\lambda)=(n-1)(g-1) \text {. }
$$

Proof. From Lemma 2.1, $\lambda_{\alpha \beta}$ satisfies the differential equation

$$
\lambda_{\alpha \beta}^{-1}\left(z_{\beta}\right) \frac{d}{d z_{\beta}} \lambda_{\alpha \beta}\left(z_{\beta}\right)+\frac{n-1}{2}\left(\frac{d z_{\alpha}}{d z_{\beta}}\right)^{-1}\left(\frac{d}{d z_{\beta}}\right)^{2} z_{\alpha}=0 .
$$

The solutions of this equation are given by

$$
\lambda_{\alpha \beta}\left(z_{\beta}\right)=\frac{e_{\alpha \beta}}{\left(d z_{\alpha} / d z_{\beta}\right)^{n-1 / 2}} \quad\left(e_{\alpha \beta} \in C\right) .
$$

Since the transition function of the canonical line bundle is defined by

$$
\kappa_{\alpha \beta}\left(z_{\beta}\right)=\left(\frac{d z_{\alpha}}{d z_{\beta}}\right)^{-1}=\left(c_{\alpha \beta} z_{\beta}+d_{\alpha \beta}\right)^{2} \quad \text { in } U_{\alpha} \cap U_{\beta},
$$

and

$$
\operatorname{deg} \kappa_{\alpha \beta}=2(g-1),
$$

it follows that

$$
\operatorname{deg}\left(\lambda_{\alpha \beta}\right)=(n-1)(g-1) .
$$

We shall now prove an analogous theorem of the Laguerre-Forsyth's basic differential invariants. 
TheOREm 2.1. Let $\mathfrak{A}=\left(U_{\alpha}, z_{\alpha}\right)$ be a projective coordinate covering of $X, \lambda \in H^{1}\left(X, \mathcal{O}^{\times}\right)$be a complex line bundle of $X$, and let $L_{n}(P \mid z)$ be a $\lambda$ related semi-canonical form. In each projective coordinate open set $U_{\alpha}$ we introduce holomorphic functions $\theta_{m, \alpha}\left(z_{\alpha}\right)(m=2,3, \cdots, n)$ by:

$$
\begin{aligned}
\theta_{m, \alpha}\left(z_{\alpha}\right)= & \frac{1}{2} \sum_{k=0}^{m-2}(-1)^{k} \frac{(m-2) ! m !(2 m-k-2) !}{(m-k-1) !(m-k) !(2 m-3) ! k !} \\
& \times\left(\frac{d}{d z_{\alpha}}\right)^{k} P_{m-k, \alpha}\left(z_{\alpha}\right) .
\end{aligned}
$$

Then

$$
\left(\theta_{m, \alpha}\left(z_{\alpha}\right)\right) \in \Gamma\left(\mathfrak{A}, \mathcal{O}\left(\kappa^{m}\right)\right)
$$

where $\kappa$ is the canonical line bundle of $X$.

Conversely if local holomorphic functions $\theta_{m, \alpha}\left(z_{\alpha}\right)$ represent an element of $\Gamma\left(X, \mathcal{O}\left(\kappa^{m}\right)\right)(m=2,3, \cdots, n)$, then there is a complex line bundle $\lambda \in$ $H^{1}\left(X, \mathcal{O}^{\times}\right)$and, if we put

$$
P_{\ell, \alpha}\left(z_{\alpha}\right)=\sum_{m=2}^{\ell}\left(\begin{array}{l}
\ell \\
m
\end{array}\right)-\frac{(\ell-1) !(2 m-1) !}{(m-1) !(m+\ell-1) !}\left(\frac{d}{d z_{\alpha}}\right)^{\ell-m} \theta_{m, \alpha}\left(z_{\alpha}\right),
$$

the semi-canonical form $L_{n}(P \mid z)$ defined in each projective coordinate open set $U_{\alpha}$ by

$$
L_{n, \alpha}\left(P_{\alpha} \mid z_{\alpha}\right)=\left(\frac{d}{d z_{\alpha}}\right)^{n}+\sum_{\ell=2}^{n}\left(\begin{array}{c}
n \\
\ell
\end{array}\right) P_{\ell, \alpha}\left(z_{\alpha}\right)\left(\frac{d}{d z_{\alpha}}\right)^{n-\ell}
$$

is a i-related semi-canonical form.

To prove Theorem 2.1, we need some lemmas

Lemma 2.2. Let $\left(\begin{array}{ll}a & b \\ c & d\end{array}\right) \in S L(2, C)$, then we have;

(i ) $\left(\frac{d}{d z}\right)^{m}\left((c z+d)^{-w} h\left(\frac{a z+b}{c z+d}\right)\right)$

$$
=\sum_{p=0}^{m}(-1)^{p}\left(\begin{array}{c}
m \\
p
\end{array}\right) \frac{[w]_{m}}{[w]_{m-p}} c^{p}(c z+d)^{-w-2 m+p}\left(\left(\frac{d}{d z}\right)^{m-p} h\right)\left(\frac{a z+b}{c z+d}\right)
$$

and

(ii) $\begin{aligned} & \left(\frac{d}{d((a z+b) /(c z+d))}\right)^{m}\left((c z+d)^{-w} h(z)\right) \\ & =\sum_{p=0}^{m}(-1)^{p}\left(\begin{array}{c}m \\ p\end{array}\right) \frac{(w)_{m}}{(w)_{m-p}} c^{p}(c z+d)^{-w+2 m-p}\left(\frac{d}{d z}\right)^{m-p} h(z)\end{aligned}$ 
where

$$
[w]_{\ell}=\left\{\begin{array}{cl}
w(w+1) \cdots(w+\ell-1) & \text { if } \ell>0 \\
1 & \text { if } \ell=0
\end{array}\right.
$$

and

$$
(w)_{\ell}=\left\{\begin{array}{cl}
w(w-1) \cdots(w-\ell+1) & \text { if } \ell>0 \\
1 & \text { if } \ell=0 .
\end{array}\right.
$$

Proof. For the proof of (ii), see ([1] Lemma 1.12).

Let us prove by (i) by induction on $m$. It is obvious for $m=0$. Assume (i) for $m$ and let us prove (i) for $m+1$. Since $a d-b c=1$

$$
\frac{d}{d z} h\left(\frac{a z+b}{c z+d}\right)=(c z+d)^{-2}\left(\frac{d}{d z} h\left(\frac{a z+b}{c z+d}\right)\right.
$$

and

$$
\begin{aligned}
\left(\frac{d}{d z}\right)^{m+1}(c z+d)^{-w} h\left(\frac{a z+b}{c z+d}\right) & \\
= & \frac{d}{d z}\left(\sum_{q=0}^{m}(-1)^{p}\left(\begin{array}{c}
m \\
p
\end{array}\right) \frac{[w]_{m}}{[w]_{m-p}} c^{p}(c z+d)^{-w-2 m+p}\left(\left(\frac{d}{d z}\right)^{m-p} h\right)\left(\frac{a z+b}{c z+d}\right)\right) \\
= & \sum_{p=0}^{m}(-1)^{p}\left(\begin{array}{c}
m \\
p
\end{array}\right) \frac{[w]_{m}}{[w]_{m-p}}(p-w-2 m) c^{p+1}(c z+d)^{p-w-2 m-1} \\
& \cdot\left(\left(\frac{d}{d z}\right)^{m-p} h\right)\left(\frac{a z+b}{c z+d}\right) \\
& +\sum_{p=0}^{m}(-1)^{p}\left(\begin{array}{l}
m \\
p
\end{array}\right) \frac{[w]_{m}}{[w]_{m-p}} c^{p}(c z+d)^{p-w-2 m-2}\left(\left(\frac{d}{d z}\right)^{m-p+1} h\right)\left(\frac{a z+b}{c z+d}\right) \\
= & \sum_{p=1}^{m}(-1)^{p}\left(\left(\begin{array}{l}
m \\
p
\end{array}\right) \frac{[w]_{m}}{[w]_{m-p}}-\left(\frac{m}{p-1}\right) \frac{[w]_{m}}{[w]_{m-p+1}}(p-1-w-2 m)\right) \\
& +(c z+d)^{-w-2 m-2}\left(\left(\frac{d}{d z}\right)^{m+1} h\right)\left(\frac{a z+b}{c z+d}\right) \\
& +(-1)^{m+1}[w]_{m+1} c^{m+1}(c z+d)^{-w-m-1} h\left(\frac{a z+b}{c z+d}\right) .
\end{aligned}
$$

By a simple calculation, we find

$$
\left(\begin{array}{c}
m \\
p
\end{array}\right) \frac{[w]_{m}}{[w]_{m-p}}-\left(\frac{m}{p-1}\right) \frac{[w]_{m}}{[w]_{m-p+1}}(p-1-w-2 m)=\left(\frac{m+1}{p}\right) \frac{[w]_{m+1}}{[w]_{m+1-\ell}} .
$$


Hence we have

$$
\begin{aligned}
& \left(\frac{d}{d z}\right)^{m+1}(c z+d)^{-w} h\left(\frac{a z+b}{c z+d}\right) \\
& \quad=\sum_{p=0}^{m+1}(-1)^{p}\left(\frac{m+1}{p}\right) \frac{[w]_{m+1}}{[w]_{m+1-p}} c^{p}(c z+d)^{-w-2(m+1)+p}\left(\left(\frac{d}{d z}\right)^{m+1-p} h\right)\left(\frac{a z+b}{c z+d}\right) .
\end{aligned}
$$

This completes the proof.

Q.E.D.

Lemma 2.3. Let $X$ be a compact Riemann surface of genus $g>1$, let $\mathfrak{U}=\left\{U_{\alpha}, z_{\alpha}\right\}$ be a projective coordinate covering of $X$ such that the transition functions are given by

$$
z_{\alpha}=\frac{a_{\alpha \beta} z_{\beta}+b_{\alpha \beta}}{c_{\alpha \beta} z_{\beta}+d_{\alpha \beta}} \quad\left(\begin{array}{ll}
a_{\alpha \beta} & b_{\alpha \beta} \\
c_{\alpha \beta} & d_{\alpha \beta}
\end{array}\right) \in S L(2, C),
$$

and let $\lambda \in H^{1}\left(X, \mathcal{O}^{\times}\right)$be a complex line bundle on $X$. Then linear differential operators $L_{n}(P \mid z)$ is a $\lambda$-related semi-canonical form if and only if coefficients $\left\{P_{m, \alpha}\left(z_{\alpha}\right)\right\}$ satisfy in each $U_{\alpha} \cap U_{\beta}$

$$
P_{2, \beta}\left(z_{\beta}\right)=\left(c_{\alpha \beta} z_{\beta}+d_{\alpha \beta}\right)^{-4} P_{2, \alpha}\left(z_{\alpha}\right)
$$

and for $m=3,4, \cdots, n$,

$$
\begin{aligned}
P_{m, \beta}\left(z_{\beta}\right)= & \left(c_{\alpha \beta} z_{\beta}+d_{\alpha \beta}\right)^{-2 m}\left(P_{m, \alpha}\left(z_{\alpha}\right)\right. \\
& +\sum_{p=1}^{m-2}(-1)^{p}\left(\begin{array}{c}
m \\
p
\end{array}\right)(m-1)(m-2) \cdots(m-p) c_{\alpha \beta}^{p} \\
& \left.\cdot\left(c_{\alpha \beta} z_{\beta}+d_{\alpha \beta}^{p}\right) P_{m-p, \alpha}\left(z_{\alpha}\right)\right) .
\end{aligned}
$$

Proof. $L_{n}(P \mid z)$ is a $\lambda^{n-1}$-related semi-canonical form if and only if in each $U_{\alpha} \cap U_{\beta}$

$$
L_{n, \beta}\left(P_{\beta} \mid z_{\beta}\right) y=\left(\frac{d z_{\alpha}}{d z_{\beta}}\right)^{n} \lambda_{\alpha \beta} L_{n, \alpha}\left(P_{\alpha} \mid z_{\alpha}\right) \lambda_{\alpha \beta}^{-1} y .
$$

Since $a_{\alpha \beta} d_{\alpha \beta}-b_{\alpha \beta} c_{\alpha \beta}=1$, we have

$$
\frac{d z_{\alpha}}{d z_{\beta}}=\frac{1}{\left(c_{\alpha \beta} z_{\beta}+d_{\alpha \beta}\right)^{2}}
$$

and by Proposition 2.1,

$$
\lambda_{\alpha \beta}\left(z_{\beta}\right)=\left(c_{\alpha \beta} z_{\beta}+d_{\alpha \beta}\right)^{n-1} e_{\alpha \beta},
$$

where $e_{\alpha \beta}$ is a complex number.

By virtue of Lemma 2.2, we have 


$$
\left(\frac{d}{d z_{\alpha}}\right)^{n} \frac{y}{\left(c_{\alpha \beta} z_{\beta}+d_{\alpha \beta}\right)^{n-1}}=\left(c_{\alpha \beta} z_{\beta}+d_{\alpha \beta}\right)^{n+1}\left(\frac{d}{d z_{\beta}}\right)^{n} y
$$

and denoting

$$
(a)_{\ell}=a(a-1) \cdots(a-\ell+1),
$$

it follows that

$$
\begin{aligned}
& \sum\left(\begin{array}{c}
n \\
\ell
\end{array}\right) P_{n-\ell, \alpha}\left(z_{\alpha}\right)\left(\frac{d}{d z_{\alpha}}\right)^{\ell} \frac{y}{\left(c_{\alpha \beta} z_{\beta}+d_{\alpha \beta}\right)^{n-1}} \\
&=\sum_{\ell=0}^{n-2}\left(\begin{array}{c}
n \\
\ell
\end{array}\right) P_{n-\ell, \alpha}\left(z_{\alpha}\right)\left(\sum_{p=0}^{\ell}(-1)^{p}\left(\begin{array}{c}
\ell \\
p
\end{array}\right) \frac{(n-1)_{\ell}}{(n-1)_{\ell-p}} c_{\alpha \beta}^{p}\right. \\
&\left.\cdot\left(c_{\alpha \beta} z_{\beta}+d_{\alpha \beta}\right)^{-p-n+2 \ell+1}\left(\frac{d}{d z_{\beta}}\right)^{\ell-p} y\right) .
\end{aligned}
$$

Comparing the coefficient of $\left(d / d z_{\beta}\right)^{\ell} y$, we obtain the desired result.

Q.E.D.

Lemma 2.4. Let $F(z)$ be any polynomial whose degree is less than $n$. Then

$$
\sum_{\ell=0}^{n}(-1)^{\ell}\left(\begin{array}{l}
n \\
\ell
\end{array}\right) F(\ell)=0 .
$$

Proof. For a non negative integer $m$ less than $n$, we introduce the polynomial $F_{m}(z)$ by

$$
\begin{aligned}
F_{m}(z) & =\left(\frac{d}{d z}\right)^{m}(1-z)^{m} \\
& =\sum_{\ell=0}^{n}(-1)^{\ell}\left(\begin{array}{c}
n \\
\ell
\end{array}\right) \ell(\ell-1) \cdots(\ell-m+1) z^{\ell-m} .
\end{aligned}
$$

Since $F_{m}(1)=0$, it follows that

$$
\sum_{\ell=0}^{n}(-1)^{\ell}\left(\begin{array}{l}
n \\
\ell
\end{array}\right) \ell(\ell-1) \cdots(\ell-m+1)=0 .
$$

We can now conclude (2.13) by induction on the degree of $F(z)$. Q.E.D.

Proof of Theorem 2.1 By virtue of Lemma 2.3, we have

$$
\begin{aligned}
\left(\frac{d}{d z_{\beta}}\right)^{\ell} P_{m, \beta}\left(z_{, j}\right)= & \left(c_{\alpha \beta} z_{\beta}+d_{\alpha \beta}\right)^{-2(m+\ell)} \sum_{p=0}^{m-2} \sum_{q=0}^{\ell}(-1)^{p+q}\left\{\begin{array}{c}
m \\
p
\end{array}\right\}\left(\begin{array}{l}
\ell \\
q
\end{array}\right) \frac{[2 m-p]_{\ell}}{[2 m-p]_{\ell-q}} \\
& \cdot c_{\alpha \beta}^{p+q}\left(c_{\alpha \beta} z_{\beta}+d_{\alpha \beta}\right)^{p+q}\left(\frac{d}{d z_{\beta}}\right)^{\ell-q} P_{m-p, \alpha}\left(z_{\alpha}\right)
\end{aligned}
$$




$$
\text { where }\left\{\begin{array}{l}
m \\
p
\end{array}\right\}=\left\{\begin{array}{cl}
1 & \text { if } p=0 \\
\left(\begin{array}{l}
m \\
p
\end{array}\right)(m-1) \cdots(m-p) & \text { if } p>0
\end{array}\right.
$$

Therefore we have;

$$
\begin{aligned}
\theta_{m, \beta}\left(z_{\beta}\right)= & \left(c_{\alpha \beta} z_{\beta}+d_{\alpha \beta}\right)^{-2 m} \sum_{\ell=0}^{m-2} \sum_{p=0}^{m-\ell-2} \sum_{q=0}^{\ell}(-1)^{p+q}\left\{\begin{array}{c}
m-\ell \\
p
\end{array}\right\}\left(\begin{array}{c}
\ell \\
q
\end{array}\right) A_{m, \ell} \\
& \cdot \frac{[2 m-2 l-p]_{\ell}}{[2 m-2 l-p]_{\ell-q}} c_{\alpha \beta}^{p+q}\left(c_{\alpha \beta} z_{\beta}+d_{\alpha \beta}\right)^{p+q}\left(\frac{d}{d z_{\beta}}\right)^{\ell-q} P_{m-p-\ell, \alpha}\left(z_{\alpha}\right)
\end{aligned}
$$

where

$$
A_{m, \ell}=\frac{1}{2}(-1)^{\ell} \frac{(m-2) ! m !(2 m-\ell-2) !}{(m-\ell-1) !(m-\ell) !(2 m-3) ! \ell !} .
$$

By a straightforward calculation we obtain; the coefficient of

$$
\begin{aligned}
\left(c_{\alpha \beta} z_{\beta}+\right. & \left.d_{\alpha \beta}\right)^{-2 m}\left(\frac{d}{d z_{\beta}}\right)^{s} P_{t}\left(z_{\alpha}\right) \text { in } \theta_{m}\left(z_{\beta}\right) \\
= & \left((-1) c_{\alpha \beta}\left(c_{\alpha \beta} z_{\beta}+d_{\alpha \beta}\right)\right)^{m-s-\ell} \sum_{\ell=s}^{m-t}\left\{\begin{array}{l}
m-\ell \\
m-\ell-t
\end{array}\right\}\left(\begin{array}{c}
\ell \\
\ell-s
\end{array}\right) \frac{[m-\ell+t]_{\ell}}{[m-\ell+t]_{s}} A_{m, \ell} \\
= & \left((-1) c_{\alpha \beta}\left(c_{\alpha \beta} z_{\beta}+d_{\alpha \beta}\right)\right)^{m-s-\ell} c(m, s, t) \sum_{\ell=s}^{m-t}(-1)^{\ell} \\
& \cdot \frac{(2 m-\ell-2) !}{(\ell-s) !(m-\ell-t) !(m-\ell+t+s-1) !}
\end{aligned}
$$

where $c(m, s, t)$ is a constant depending sololy on $m, s, t$.

Since

$$
\begin{gathered}
\sum_{\ell=s}^{m-t}(-1)^{\ell} \frac{(2 m-\ell-2) !}{(\ell-s) !(m-\ell-t) !(m-\ell+t+s-1) !} \\
=\frac{1}{a !} \sum_{\ell=0}^{a}(-1)^{\ell}\left(\begin{array}{l}
a \\
\ell
\end{array}\right) \frac{(2 s+2 a+2 t-\ell-2) !}{(2 s+a+2 t-\ell-1) !} \\
\text { where } a=m-t-s,
\end{gathered}
$$

and since $(2 s+2 a+2 t-\ell-2) ! /(2 s+a+2 t-\ell-1) !$ is a polynomial $F(\ell)$ of degree $a-1$ with respect to $\ell$, we have

$$
\begin{aligned}
\sum_{\ell=s}^{m-t}(-1)^{\ell} & \frac{(2 m-\ell-2) !}{(\ell-s) !(m-\ell-t) !(m-\ell+t+s-1) !} \\
& =\frac{1}{a !} \sum_{\ell=0}^{a}(-1)^{\ell}\left(\begin{array}{l}
a \\
\ell
\end{array}\right) F(\ell) .
\end{aligned}
$$


By virtue of Lemma 2.4, if $a>0$ then it follows that

$$
\frac{1}{a !} \sum_{\ell=0}^{a}(-1)^{\ell}\left(\begin{array}{l}
a \\
\ell
\end{array}\right) F(\ell)=0 \text {. }
$$

This means that the coefficient of $\left(c_{\alpha \beta} z_{\beta}+d_{\alpha \beta}\right)^{-2 m}\left(d / d z_{\beta}\right)^{s} P_{t, \alpha}\left(z_{\alpha}\right)(m>t+s)$ in $\theta_{m, \beta}\left(z_{\beta}\right)$ is zero.

We can now conclude

$$
\theta_{m, \alpha}\left(z_{\alpha}\right)=\left(c_{\alpha \beta} z_{\beta}+d_{\alpha \beta}\right)^{2 m} \theta_{m, \beta}\left(z_{\beta}\right) .
$$

Conversely if we put,

$$
P_{\ell, \alpha}\left(z_{\alpha}\right)=\sum_{m=2}^{\ell}\left(\begin{array}{l}
\ell \\
m
\end{array}\right) \frac{(\ell-1) !(2 m-1) !}{(m-1) !(m+\ell-1) !}\left(\frac{d}{d z_{\alpha}}\right)^{\ell-m} \theta_{m, \alpha}\left(z_{\alpha}\right),
$$

we can verify that the semi-canonical form defined by (2.18) is $\lambda$-related if and only if the equation (2.14) holds. But we have shown that in the equation (2.14) there are no terms with $p+q>0$, hence the right hand side of (2.14) is equal to

$$
\left(c_{\alpha \beta} z_{\beta}+d_{\alpha \beta}\right)^{-2 m} \theta_{m, \alpha}\left(z_{\alpha}\right)=\theta_{m, \beta}\left(z_{\beta}\right) .
$$

This completes the proof.

Q.E.D.

Theorem 2.2. Let $X$ be a compact Riemann surface of genus $g>1$ and for a projective coordinate covering $\mathfrak{U}=\left\{U_{\alpha}, z_{\alpha}\right\}$, let $\left\{\theta_{m, \alpha}\left(z_{\alpha}\right)\right\} \in \Gamma\left(\mathfrak{A}, \mathcal{O}\left(\kappa^{m}\right)\right)$ $(m=2,3, \cdots, n)$. In each coordinate open set $U_{\alpha}$, consider the differential equation

$$
\left(\frac{d}{d z_{\alpha}}\right)^{n} y+\sum_{\ell=2}^{n}\left(\begin{array}{c}
n \\
\ell
\end{array}\right) P_{\ell, \alpha}\left(z_{\alpha}\right)\left(\frac{d}{d z_{\alpha}}\right)^{n-\ell} y=0
$$

where $\left\{P_{\ell, \alpha}\left(z_{\alpha}\right)\right\}$ are defined by

$$
P_{\ell, \alpha}\left(z_{\alpha}\right)=\sum_{m=2}^{\ell}\left(\begin{array}{c}
\ell \\
m
\end{array}\right) \frac{(\ell-1) !(2 m-1) !}{(m-1) !(m+\ell-1) !}\left(\frac{d}{d z_{\alpha}}\right)^{\ell-m} \theta_{m, \alpha}\left(z_{\alpha}\right) .
$$

Selecting $n$ linearly independent holomorphic solutions $\varphi_{1, \alpha}\left(z_{\alpha}\right) \cdots \varphi_{n, \alpha}\left(z_{\alpha}\right)$ of the differential equation (2.15), introduce the vector-valued functions in each coordinate open set $U_{\alpha}$ as follows

$$
\varphi_{\alpha}\left(z_{\alpha}\right)=\left(\begin{array}{c}
\varphi_{1, \alpha}\left(z_{\alpha}\right) \\
\varphi_{2, \alpha}\left(z_{\alpha}\right) \\
\vdots \\
\varphi_{n, \alpha}\left(z_{\alpha}\right)
\end{array}\right)
$$


hen in each intersection $U_{\alpha} \cap U_{\beta}$ there is a unique matrix $\rho_{\alpha \beta} \in S L(n, C)$ ıch that

$$
\begin{aligned}
\varphi_{\alpha}\left(z_{\alpha}\right)=\rho_{\alpha \beta}\left(c_{\alpha \beta} z_{\beta}+d_{\alpha \beta}\right)^{1-n} \varphi_{\beta}\left(z_{\beta}\right) \\
\operatorname{det}\left[\varphi_{\alpha}\left(z_{\alpha}\right) \frac{d}{d z_{\alpha}} \varphi_{\alpha}\left(z_{\alpha}\right), \cdots,\left(\frac{d}{d z_{\alpha}}\right)^{n-1} \varphi_{\alpha}\left(z_{\alpha}\right)\right]=c_{\alpha} \\
\text { where } c_{\alpha} \text { is a non zero constant. }
\end{aligned}
$$

'onversely if holomorphic vector valued functions $\left\{\varphi_{\alpha}\left(z_{\alpha}\right)\right\}$ defined in each rojective open set $U_{\alpha}$ satisfy (2.16), then $\left\{\theta_{m, \alpha}\left(z_{\alpha}\right)\right\}$ defined by (2.6) is an 'ement of $\Gamma\left(u, \mathcal{O}\left(\kappa^{m}\right)\right)$.

Proof. By Theorem 2.1, the semi-canonical form $L_{n}(P \mid z)$ is a $\lambda$-related ifferential operator. Therefore if $\varphi_{1, \alpha}\left(z_{\alpha}\right), \cdots, \varphi_{n, \alpha}\left(z_{\alpha}\right)$ are $n$ linearly indeendent solutions of the differential equation (2.15), $\lambda_{\beta, \alpha} \varphi_{1, \alpha}\left(z_{\alpha}\right), \cdots, \lambda_{\beta, \alpha} \varphi_{n, \alpha}\left(z_{\alpha}\right)$ re linearly independent solutions of (2.15) in $U_{\beta}$. Hence there is a non ingular $n \times n$ matrix $\rho_{\alpha \beta}$ and $\left\{\varphi_{\alpha}\left(z_{\alpha}\right)\right\}$ satisfy in $U_{\alpha} \cap U_{\beta}$

$$
\varphi_{\alpha}\left(z_{\alpha}\right)=\rho_{\alpha \beta}\left(c_{\alpha \beta} z_{\beta}+d_{\alpha \beta}\right)^{1-n} \varphi_{\beta}\left(z_{\beta}\right) .
$$

ince $L_{n}(P \mid z)$ is a semi-canonical form, the Wronskian of functions $\varphi_{1, \alpha}\left(z_{\alpha}\right)$, ${ }_{2, \alpha}\left(z_{\alpha}\right), \cdots, \varphi_{n, \alpha}\left(z_{\alpha}\right)$ is constant. Hence the matrix $\rho_{\alpha \beta}$ is contained in $i L(n, C)$. The converse is obvious from Theorem 2.1 .

Q.E.D.

\section{The transvectant}

In this paragraph we shall introduce differential operators called the ransvectants. The transvectan' $\mathrm{c}$ is the one of basic methods to create ew covariants from given covariants and is known since 19-th century n the classical invariant theory, we shall now generalize the classical ransvectants.

Let $\mathfrak{A}=\left(U_{\alpha}, z_{\alpha}\right)$ be a projective coordinate covering of a Riemann urface $X$ such that the coordinate transition functions are given by

$$
z_{\alpha}=\frac{a_{\alpha \beta} z_{\beta}+b_{\alpha \beta}}{c_{\alpha \beta} z_{\beta}+d_{\alpha \beta}}, \quad \text { with } \quad \sigma_{\alpha \beta}=\left(\begin{array}{l}
a_{\alpha \beta}, b_{\alpha \beta} \\
c_{\alpha \beta}, d_{\alpha \beta}
\end{array}\right) \in S L(2, C) .
$$

Let $p$ be a point in $X$, and consider in various neighborhoods $U_{\alpha}$ of he point $n_{1} \times n_{2}$ matrix valued holomorphic functions $f_{\alpha}\left(z_{\alpha}\right)$ such that, if he point $p$ is contained in $U_{\alpha} \cap U_{\beta},\left\{f_{\alpha}\left(z_{\alpha}\right)\right\}$ satisfy;

$$
\begin{aligned}
& f_{\alpha}\left(z_{\alpha}\right)=\left(c_{\alpha \beta} z_{\beta}+d_{\alpha \beta}\right)^{-m_{1}} A_{\alpha \beta} f_{\beta}\left(z_{\beta}\right) \\
& \text { where } m_{1} \text { is a integer and } A_{\alpha \beta} \in G L(n, C) .
\end{aligned}
$$


Similarly let $\left\{g_{\alpha}\left(z_{\alpha}\right)\right\}$ be $n_{3} \times n_{2}$ matrix valued holomorphic functions such that, in a neighborhood $U_{\alpha} \cap U_{\beta}$ of $p,\left\{g_{\alpha}\left(z_{\alpha}\right)\right\}$ satisfy

$$
g_{\alpha}\left(z_{\alpha}\right)=\left(c_{\alpha \beta} z_{\beta}+d_{\alpha \beta}\right)^{-m_{2}} B_{\alpha \beta} g_{\beta}\left(z_{\beta}\right)
$$

where $m_{2}$ is a integer and $B_{\alpha \beta} \in G L\left(n_{2}, C\right)$.

Definition 3.1. Denoting $(m)_{k} k \in\{0,1,2, \cdots\}$ by

$$
(m)_{k}=\left\{\begin{array}{cl}
(m(m-1) \cdots(m-k+1) & \text { if } k>0 \\
1 & \text { if } k=0,
\end{array}\right.
$$

assume $\left(m_{1}\right)_{r}\left(m_{2}\right)_{r} \neq 0$. Then the $r$-th transvectant $\left\langle f_{\alpha}\left(z_{\alpha}\right), g_{\alpha}\left(z_{\alpha}\right)\right\rangle^{r}$ of $f_{\alpha}\left(z_{\alpha}\right)$ and $g_{\alpha}\left(z_{\alpha}\right)$ is defined as follows

$$
\begin{aligned}
\left\langle f_{\alpha}\left(z_{\alpha}\right),\right. & \left.g_{\alpha}\left(z_{\alpha}\right)\right\rangle^{r}\left(z_{\alpha}\right) \\
= & \sum_{l=0}^{r}(-1)^{\ell}\left(\begin{array}{c}
r \\
\ell
\end{array}\right) \frac{1}{\left(m_{1}\right)_{\ell}\left(m_{2}\right)_{r-\ell}}\left(\frac{d}{d z_{\alpha}}\right)^{\ell} f_{\alpha}\left(z_{\alpha}\right)\left(\frac{d}{d z_{\alpha}}\right)^{n-\ell}{ }^{t} g_{\alpha}\left(z_{\alpha}\right), \\
& \text { where }{ }^{t}() \text { stands for the transposed matrix } .
\end{aligned}
$$

Proposition 3.1. Suppose matrix valued functions $f_{\alpha}\left(z_{\alpha}\right), g_{\alpha}\left(z_{\alpha}\right)$ satisfy (3.1), (3.2), and if $\left(m_{1}\right)_{r}\left(m_{2}\right)_{r} \neq 0$, then the $r$-th transvectants $\left\langle f_{\alpha}\left(z_{\alpha}\right), g_{\alpha}\left(z_{\alpha}\right)\right\rangle^{r}$ satisfy the following transformation relation

$$
\left\langle f_{\alpha}\left(z_{\alpha}\right), g_{\alpha}\left(z_{\alpha}\right)\right\rangle^{r}=\left(c_{\alpha \beta} z_{\beta}+d_{\alpha \beta}\right)^{2 r-\left(m_{1}+m_{2}\right)} A_{\alpha \beta}\left\langle f_{\beta}\left(z_{\beta}\right), g_{\beta}\left(z_{\beta}\right)\right\rangle^{r t} B_{\alpha \beta} .
$$

Proof. By virtue of (ii) in Lemma 2.2, we have

$$
\begin{aligned}
& \frac{1}{\left(m_{1}\right)^{\ell}}\left(\frac{d}{d z_{\alpha}}\right)^{\ell} f_{\alpha}\left(z_{\alpha}\right) \\
& \quad=A_{\alpha \beta}\left(c_{\alpha \beta} z_{\beta}+d_{\alpha \beta}\right)^{2 \ell-m_{1}} \sum_{p=0}^{\ell}(-1)^{\ell}\left(\begin{array}{c}
\ell \\
p
\end{array}\right) \frac{\left(d / d z_{\beta}\right)^{\ell-p} f_{\beta}\left(z_{\beta}\right)}{\left(m_{1}\right)_{\ell-p}} c_{\alpha \beta}^{p}\left(c_{\alpha \beta} z_{\beta}+d_{\alpha \beta}\right)^{-p},
\end{aligned}
$$

and

$$
\begin{aligned}
& -\frac{1}{\left(m_{2}\right)^{\ell}}\left(\frac{d}{d z_{\alpha}}\right)^{\ell} g_{\alpha}\left(z_{\alpha}\right) \\
& \quad=B_{\alpha \beta}\left(c_{\alpha \beta} z_{\beta}+d_{\alpha \beta}\right)^{2 \ell-m_{2}} \sum_{p=0}^{\ell}(-1)^{\ell}\left(\begin{array}{c}
\ell \\
p
\end{array}\right) \frac{\left(d / d z_{\beta}\right)^{\ell-p} g_{\beta}\left(z_{\beta}\right)}{\left(m_{2}\right)_{\ell-p}} c_{\alpha \beta}^{p}\left(c_{\alpha \beta} z_{\beta}+d_{\alpha \beta}\right)^{-p} .
\end{aligned}
$$

Hence, putting

$$
\begin{aligned}
M(f, g)= & \left(\sum_{\ell=0}^{r}(-1)^{\ell}\left(\begin{array}{c}
r \\
\ell
\end{array}\right)\left(\sum_{p=0}^{\ell}(-1)^{p}\left(\begin{array}{c}
\ell \\
p
\end{array}\right) \frac{\left(d / d z_{\beta}\right)^{\ell-p} f_{\beta}\left(z_{\beta}\right)}{\left(m_{1}\right)_{\ell-p}}\left(\frac{c_{\alpha \beta}}{c_{\alpha \beta} z_{\beta}+d_{\alpha \beta}}\right)^{p}\right)\right. \\
& \cdot\left(\sum_{q=0}^{r-\ell}(-1)^{q}\left(\begin{array}{c}
r-\ell \\
q
\end{array}\right) \frac{\left(d / d z_{\beta}\right)^{r-\ell-q} g_{\beta}\left(z_{\beta}\right)}{\left(m_{2}\right)_{r-\ell-q}}\left(\frac{c_{\alpha \beta}}{c_{\alpha \beta} z_{\beta}+d_{\alpha \beta}}\right)^{q}\right),
\end{aligned}
$$


we obtain

$$
\left\langle f_{\alpha}\left(z_{\alpha}\right), g_{\alpha}\left(z_{\alpha}\right)\right\rangle^{r}=\left(c_{\alpha \beta} z_{\beta}+d_{\alpha \beta}\right)^{2 r-\left(m_{1}+m_{2}\right)} A_{\alpha \beta} M(f, g){ }^{t} B_{\alpha \beta} .
$$

We shall show that

$$
M(f, g)=\left\langle f_{\beta}\left(z_{\beta}\right), g_{\beta}\left(z_{\beta}\right)\right\rangle^{r} .
$$

Putting $\ell-p=j, r-\ell-q=k$ and $p+q=s$, denote $a(j, k, s)$ by $a(j, k, s)=$ the coefficient of $(-1)^{s} \frac{\left(d / d z_{\beta}\right)^{j} f_{\beta}\left(z_{\beta}\right)\left(d / d z_{\beta}\right)^{k t} g_{\beta}\left(z_{\beta}\right)}{\left(m_{1}\right)_{j}\left(m_{2}\right)_{k} j ! k !}\left(\frac{c_{\alpha \beta}}{c_{\alpha \beta} z_{\beta}+d_{\alpha \beta}}\right)^{s}$ in $M(f, g)$.

Then we find

$$
a(j, k, s)=\left\{\begin{array}{cl}
(-1)^{\imath} r ! & \text { if } s=0 \\
0 & \text { if } s>0
\end{array}\right.
$$

This shows

$$
M(f, g)=\left\langle f_{\beta}\left(z_{\beta}\right), g_{\beta}\left(z_{\beta}\right)\right\rangle^{r} . \quad \text { Q.E.D. }
$$

By virtue of (ii) in Lemma 2.2, the following proposition is clear.

Proposition 3.2. If matrix valued holomorphic functions $\left\{f_{\alpha}\left(z_{\alpha}\right)\right\}$ satisfy the relation (3.1) in each $U_{\alpha} \cap U_{\beta}$, then matrix valued functions $\left(d / d z_{\alpha}\right)^{m_{1+1}} f_{\alpha}\left(z_{\alpha}\right)$ satisfy in $U_{\alpha} \cap U_{\beta}$;

$$
\left(\frac{d}{d z_{\alpha}}\right)^{m_{1}+1} f_{\alpha}\left(z_{\alpha}\right)=\left(c_{\alpha \beta} z_{\beta}+d_{\alpha \beta}\right)^{m_{1}+2} A_{\alpha \beta} \quad\left(\frac{d}{d z_{\beta}}\right)^{m_{1}+1} f_{\beta}\left(z_{\beta}\right) .
$$

\section{$\S 4$. The Eichler cohomology groups}

We shall review some basic facts and definitions about Eichler cohomology groups associated to the given projective structure on the Riemann surface $X$. Let $\mathfrak{A}=\left\{U_{\alpha}, z_{\alpha}\right\}$ be a projective coordinate covering of $X$ such that the coordinate transition functions are given by

$$
z_{\alpha}=\frac{a_{\alpha \beta} z_{\beta}+b_{\alpha \beta}}{c_{\alpha \beta} z_{\beta}+d_{\alpha \beta}} \quad\left(\begin{array}{ll}
a_{\alpha \beta} & b_{\alpha \beta} \\
c_{\alpha \beta} & d_{\alpha \beta}
\end{array}\right) \in S L(2, C) \text { in } U_{\alpha} \cap U_{\beta}
$$

and let $\lambda \in H^{1}\left(X, \mathcal{O}^{\times}\right)$be a complex line bundle defined by transition functions

$$
\lambda_{\alpha \beta}\left(z_{\beta}\right)=c_{\alpha \beta} z_{\beta}+d_{\alpha \beta} .
$$

For any integer $n \geqq 0$ consider the subsheaf $P_{n}\left(\lambda^{-n}\right) \subset \mathcal{O}\left(\lambda^{-n}\right)$ consisting 
of holomorphic sections of $\lambda^{-n}$ which are polynomials of degree $\leqq n$. If $p \in U_{\alpha}$ is a point of $X$ and $f \in \mathcal{O}\left(\lambda^{-n}\right)_{p}$ is a germ of section at $p$, then $f$ is represented by a holomorphic function $f_{\alpha}\left(z_{\alpha}\right)$, and in $U_{\alpha} \cap U_{\beta}\left\{f_{\alpha}\left(z_{\alpha}\right)\right\}$ are related by

$$
f_{\beta}\left(z_{\beta}\right)=\left(c_{\alpha \beta} z_{\beta}+d_{\alpha \beta}\right)^{n} f_{\alpha}\left(z_{\alpha}\right) .
$$

By Proposition 3.1, we have an exact sequence of sheaves

$$
0 \longrightarrow P_{n}\left(\lambda^{-n}\right) \longrightarrow \mathcal{O}\left(\lambda^{-n}\right) \stackrel{d^{n+1}}{\longrightarrow} \mathcal{O}\left(\lambda^{n+2}\right) \longrightarrow 0 \quad \text { for any } n \geqq 0 .
$$

From this exact sequence 4.2 , we obtain for any integer $n \geqq 0$ an exact sequence of complex vector spaces

$$
0 \longrightarrow \Gamma\left(X, \mathcal{O}\left(\kappa^{n+1} \lambda^{-n}\right)\right) \stackrel{\delta^{*}}{\longrightarrow} H^{1}\left(X, P_{n}\left(\lambda^{-n}\right)\right) \stackrel{\nu^{*}}{\longrightarrow} \Gamma\left(X, \mathcal{O}\left(\kappa^{n+1} \lambda^{-n}\right)\right)^{*} \longrightarrow 0,
$$

where $\Gamma(-)^{*}$ stands for the dual vector space to $\Gamma(-)$. The groups $H^{1}\left(X, P_{n}\left(\lambda^{-n}\right)\right)$ are the Eichler cohomology groups of the Riemann surface $X$.

\section{§. The monodromy representations}

We shall investigate flat vector bundles coming from monodromy representations of the fundamental group of $X$.

Let $\left\{\varphi_{\alpha}\left(z_{\alpha}\right)\right\}$ be the holomorphic vector functions satisfying (2.16) in $U_{\alpha} \cap U_{\beta}$, and denote the Wronskian matrix of $\varphi_{\alpha}\left(z_{\alpha}\right)$ by $F_{\alpha}\left(z_{\alpha}\right)$ :

$$
F_{\alpha}\left(z_{\alpha}\right)=\left[\varphi_{\alpha}\left(z_{\alpha}\right),-\frac{d}{d z_{\alpha}} \varphi_{\alpha}\left(z_{\alpha}\right), \cdots,\left(\frac{d}{d z_{\alpha}}\right)^{n-1} \varphi_{\alpha}\left(z_{\alpha}\right)\right]
$$

If $p$ in a point of $U_{\alpha} \cap U_{\beta}$, there is a holomorphic vector valued function $\Phi_{\alpha \beta}(p)$ such that,

$$
\begin{aligned}
& F_{\alpha}(p) \Phi_{\alpha \beta}(p)=\rho_{\alpha \beta} F_{\beta}(p) \quad \text { for } p \in U_{\alpha} \cap U_{\beta} \text {. } \\
& \Phi_{\alpha \beta}\left(z_{\beta}\right)=\left(\begin{array}{cccc}
\lambda_{\alpha \beta}^{n-1}\left(z_{\beta}\right) & & & \\
& \lambda_{\alpha \beta}^{n-3}\left(z_{\beta}\right) & & * \\
0 & & \ddots & \\
& & & \lambda_{\alpha \beta}^{1-n}\left(z_{\beta}\right)
\end{array}\right) .
\end{aligned}
$$

Thus $\left\{\Phi_{\alpha \beta}(p)\right\}$ are considered cocycles as describing a holomorphic vector bundle $\Phi$, and flat vector bundles $\left\{\rho_{\alpha \beta}\right\}$ are analytically equivalent to $\Phi$.

We introduce vector valued functions ${ }^{t} \varphi_{\alpha}^{*}\left(\boldsymbol{z}_{\alpha}\right)=\left(\varphi_{\alpha, 1}^{*}\left(\boldsymbol{z}_{\alpha}\right), \cdots, \varphi_{\alpha, n}^{*}\left(\boldsymbol{z}_{\alpha}\right)\right)$ in each coordinate open set $\left(U_{\alpha}, z_{\alpha}\right)$ by 


$$
{ }^{t} \varphi^{*}\left(z_{\alpha}\right)=\text { the } n \text {-th row of } F_{\alpha}\left(z_{\alpha}\right)^{-1} .
$$

$\left\{\varphi_{\alpha}^{*}\left(z_{\alpha}\right)\right\}$ satisfy in each intersection $U_{\alpha} \cap U_{\beta}$

$$
\varphi_{\alpha}^{*}\left(z_{\alpha}\right)=\lambda_{\alpha \beta}^{n-1}\left(z_{\beta}\right)\left({ }^{t} \rho_{\alpha \beta}^{-1}\right) \varphi_{\beta}^{*}\left(z_{\beta}\right) .
$$

Let

$$
\begin{aligned}
\theta_{2, i}(z) & =\left\{\theta_{2, i, \alpha}\left(z_{\alpha}\right)\right\} & & \left(1 \leqq i \leqq \operatorname{dim} \Gamma\left(X, \mathcal{O}\left(\kappa^{2}\right)\right)\right. \\
\theta_{3, j}(z) & =\left\{\theta_{3, j, \alpha}\left(z_{\alpha}\right)\right\} & & \left(1 \leqq j \leqq \operatorname{dim} \Gamma\left(X, \mathcal{O}\left(\kappa^{3}\right)\right)\right. \\
& \vdots & & \\
\theta_{n, j}(z) & =\left\{\theta_{n, k, \alpha}\left(z_{\alpha}\right)\right\} & & \left(1 \leqq k \leqq \operatorname{dim} \Gamma\left(X, \mathcal{O}\left(\kappa^{n}\right)\right)\right.
\end{aligned}
$$

be a basis of the complex vector space $\Gamma\left(X, \mathcal{O}\left(\kappa^{2}\right)\right) \oplus \cdots \oplus\left(X, \mathcal{O}\left(\kappa^{n}\right)\right)$, and let $\left(\theta_{2}(z), \cdots, \theta_{n}(z)\right)$ be a point in the vector space $\oplus_{m=2}^{n} \Gamma\left(X, \mathcal{O}\left(\kappa^{m}\right)\right)$. We shall introduce local coordinate $\left\{\cdots, t_{\iota}^{(2)}, \cdots, t_{j}^{(3)}, \cdots, t_{i}^{(n)}, \cdots\right\}$ at the point $\left(\theta_{2}(z), \cdots, \theta_{n}(z)\right)$ in $\oplus_{m=2}^{n} \Gamma\left(X, \mathcal{O}\left(\kappa^{m}\right)\right)$ by

$$
\left\{\cdots, t_{i}^{(2)}, \cdots, t_{j}^{(3)}, \cdots, t_{k}^{(n)}, \cdots\right\} \longrightarrow\left\{\theta_{2}(z, t), \theta_{3}(z, t), \cdots, \theta_{n}(z, t)\right\}
$$

where $t_{\ell}^{(m)}(2 \leqq m \leqq n)$ are complex numbers such that $\left|t_{\ell}^{(m)}\right|$ are small enough, and

$$
\theta_{m}(z, t)=\theta_{m}(z)+{ }^{\operatorname{dim} \Gamma\left(X_{\ell=1}^{X, O(A m))}\right.} t_{\ell}^{(m)} \theta_{m, \ell} \quad(2 \leqq m \leqq n) .
$$

Let $\left\{\varphi_{\alpha}\left(z_{\alpha}, t\right)\right\}$ (resp. $\varphi_{\alpha}\left(z_{\alpha}\right)$ ) be vector valued holomorphic functions corresponding to the point $\left(\theta_{2}(z, t), \cdots, \theta_{n}(z, t)\right.$ ) (resp. $\left(\theta_{2}(z), \cdots, \theta_{n}(z)\right)$ in the vector space $\oplus_{m=2}^{n} \Gamma\left(X, \mathcal{O}\left(\kappa^{m}\right)\right)$ by Theorem 2.2. Then in each intersection $U_{\alpha} \cap U_{\beta}$ vector valued holomorphic functions $\left\{\varphi_{\alpha}\left(z_{\alpha}, t\right)\right\}$ satisfy;

$$
\begin{aligned}
& \varphi_{\alpha}\left(z_{\alpha}, t\right)=\left(c_{\alpha \beta} z_{\beta}+d_{\alpha \beta}\right)^{1-n} \rho_{\alpha \beta}(t) \varphi_{\beta}\left(z_{\beta}, t\right) \\
& \left.\varphi_{\alpha}\left(z_{\alpha}, t\right)\right|_{t=0}=\varphi_{\alpha}\left(z_{\alpha}\right),
\end{aligned}
$$

where $\rho_{\alpha \beta}(t) \in S L(n, C)$, and $\left.\rho_{\alpha \beta}(t)\right|_{t=0}=\rho_{\alpha \beta}$.

It is easy to verify that $\rho_{\alpha \beta}(t)$ varies complex analytically with the choice of the parameter $t=\left(\cdots t_{\ell}^{(m)} \cdots\right)$.

We consider semi-canonical forms $L_{\alpha}\left(P_{\alpha}\left(z_{\alpha}, t\right) \mid z\right)$;

$$
L_{\alpha}\left(P_{\alpha}\left(z_{\alpha}, t\right) z_{\alpha}\right)=\left(\frac{d}{d z_{\alpha}}\right)^{n}+\sum_{\ell=2}^{n}\left(\begin{array}{c}
n \\
\ell
\end{array}\right) P_{\ell, \alpha}\left(z_{\alpha}, t\right)\left(\frac{d}{d z_{\alpha}}\right)^{n-\ell},
$$

corresponding to an element $\left(\theta_{2}(z, t), \cdots, \theta_{n}(z, t)\right) \in \oplus_{m=2}^{n} \Gamma\left(X, \mathcal{O}\left(\kappa^{m}\right)\right)$, where $P_{\ell, \alpha}\left(z_{\alpha}, t\right)$ are defined by 


$$
\begin{aligned}
P_{\ell, \alpha}\left(z_{\alpha}, t\right)= & \sum_{j} \sum_{m=2}^{\ell}\left(\begin{array}{c}
\ell \\
m
\end{array}\right) \frac{(\ell-1) !(2 m-1) !}{(m-1) !(m+\ell-1) !}\left(\frac{d}{d z_{\alpha}}\right)^{\ell-m} \\
& \cdot\left(\theta_{m, \alpha}\left(z_{\alpha}\right)+t_{m j} \theta_{m j, \alpha}\left(z_{\alpha}\right)\right)
\end{aligned}
$$

and

$$
\theta_{m}(z, t)=\theta_{m}(z)+\sum t_{m j} \theta_{m j}(z) \quad \text { for } m=2,3, \cdots, n .
$$

In each coordinate neighborhood $U_{\alpha}$ with local coordinate $z_{\alpha}$, select a point $p_{\alpha}$, and consider the differential equations

$$
L_{\alpha}\left(P_{\alpha}\left(z_{\alpha}, t\right), z_{\alpha}\right) y\left(z_{\alpha}, t\right)=0
$$

under the initial conditions;

$$
F_{\alpha, t}(p)=\left(\begin{array}{lll}
1 & & 0 \\
& \ddots & \\
0 & & 1
\end{array}\right)
$$

where $p$ is a point in $U_{\alpha}$ and $F_{\alpha, t}\left(z_{\alpha}\right)$ is the Wronskian matrix of the differential equation (5.11).

If $U_{\alpha}$ and $W$ both are chosen sufficiently small, there are unique holomorphic solutions $\varphi_{\alpha}\left(z_{\alpha}, t\right)$ satisfying (5.7).

By an easy calculation, we can verify that the vector valued functions $\left.\left(\partial / \partial t_{m j}\right) \varphi_{\alpha}\left(z_{\alpha}, t\right)\right|_{t=0}$ satisfy the non-linear differential equations:

$$
\begin{gathered}
\left(\frac{d}{d z_{\alpha}}\right)^{n} y+\sum_{\ell=2}^{n}\left(\begin{array}{c}
n \\
\ell
\end{array}\right) P_{\ell, \alpha}\left(z_{\alpha}\right)\left(\frac{d}{d z_{\alpha}}\right)^{n-\ell} y \\
+\sum_{\ell=2}^{n}\left(\begin{array}{c}
n \\
\ell
\end{array}\right)\left(\begin{array}{c}
\ell \\
m
\end{array}\right) \frac{(\ell-1) !(2 m-1) !}{(m-1) !(m+\ell-1) !}\left(\frac{d}{d z_{\alpha}}\right)^{\ell-m} \\
\cdot \theta_{m j \alpha}\left(z_{\alpha}\right)\left(\frac{d}{d z_{\alpha}}\right)^{n-\ell} y=0 .
\end{gathered}
$$

Definition 5.1. Lel $\mathfrak{U}=\left\{\left(U_{\alpha}, z_{\alpha}\right)\right\}$ be a projective coordinate covering of a compact Riemann surface $X$, let $\left\{q_{\alpha}\left(z_{\alpha}\right)\right\} \in \Gamma\left(\mathscr{X}, \mathcal{O}\left(\kappa^{m}\right)\right)(m=2,3, \cdots, n)$ and let $\left\{\varphi_{\alpha}\left(z_{\alpha}\right)\right\}$ be vector valued holomorphic functions satisfying the relation (2.16) in each $U_{\alpha} \cap U_{\beta}$. We define the matrix valued holomorphic differential 1-forms $\theta_{\alpha}^{[\varphi, q]}\left(z_{\alpha}\right)$ in each coordinate open set $U_{\alpha}$ as follows

$$
\begin{aligned}
\theta_{\alpha}^{[\varphi, q]}\left(z_{\alpha}\right)= & \sum_{\ell=m}^{n}\left(\begin{array}{l}
n \\
\ell
\end{array}\right)\left(\begin{array}{c}
\ell \\
m
\end{array}\right) \frac{(\ell-1) !(2 m-1) !}{(m-1) !(m+\ell-1) !}\left(\frac{d}{d z_{\alpha}}\right)^{n-\ell} \\
& \cdot \varphi_{\alpha}\left(z_{\alpha}\right)\left(\frac{d}{d z_{\alpha}}\right)^{\ell-m} q_{\alpha}\left(z_{\alpha}\right)^{t} \varphi_{\alpha}^{*}\left(z_{\alpha}\right) d z_{\alpha} .
\end{aligned}
$$


Lemma 5.1. If $p$ is a point of $U_{\alpha} \cap U_{\beta}, n \times n$ matrix valued holomorphic 1-forms $\left\{\theta_{\alpha}^{[\varphi, q]}\left(z_{\alpha}\right)\right\}$ satisfy in $U_{\alpha} \cap U_{\beta}$

$$
\theta_{\alpha}^{[\omega, q]}\left(z_{\alpha}(p)\right)=A_{\alpha}\left(\rho_{\alpha \beta}\right) \theta_{\beta}^{[\varphi, q]}\left(z_{\beta}(p)\right) .
$$

Proof. Denoting the vector valued holomorphic function $A_{\alpha}\left(z_{\alpha}\right)$ by

$$
A_{\alpha}\left(z_{\alpha}\right)=\sum_{\ell=m}^{n}\left(\begin{array}{c}
n \\
\ell
\end{array}\right)\left(\begin{array}{c}
\ell \\
m
\end{array}\right) \frac{(\ell-1) !(2 m-1) !}{(m-1) !(m+\ell-1) !}\left(\frac{d}{d z_{\alpha}}\right)^{n-\ell} \varphi_{\alpha}\left(z_{\alpha}\right)\left(\frac{d}{d z_{\alpha}}\right)^{\ell-m} q_{\alpha}\left(z_{\alpha}\right) .
$$

A straightforward computation shows that

$$
A_{\alpha}\left(z_{\alpha}\right)=\left(\begin{array}{c}
n \\
m
\end{array}\right) \frac{(n-1) !}{(m-1) !}\left\langle\varphi_{\alpha}\left(z_{\alpha}\right), q_{\alpha}\left(z_{\alpha}\right)\right\rangle^{n-m}
$$

where $\langle,\rangle^{n-m}$ stands for $n-m$-th transvectant.

By a property of the transvection, $\left\{A_{\alpha}\left(z_{\alpha}\right)\right\}$ satisfy in $U_{a} \cap U_{\hat{\beta}}$

$$
A_{\alpha}\left(z_{\alpha}\right)=\rho_{\alpha \beta}\left(c_{\alpha \beta} z_{\beta}+d_{\alpha \beta}\right)^{n+1} A_{\beta}\left(z_{\beta}\right) .
$$

Applying the relation (5.5), it follows that

$$
A_{\alpha}\left(z_{\alpha}\right)^{t} \varphi_{\alpha}\left(z_{\alpha}\right)=\rho_{\alpha \beta} \kappa_{\alpha \beta}\left(z_{\beta}\right) A_{\beta}\left(z_{\beta}\right)^{t} \varphi_{\beta}\left(z_{\beta}\right) \rho_{\alpha \beta}^{-1} .
$$

This completes the proof.

Q.E.D.

Lemma 5.2. Let $p$ be a non negative integer, then

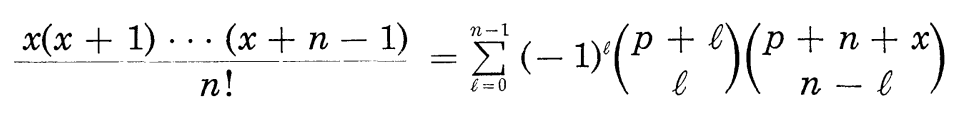

where

$$
\left(\begin{array}{l}
x \\
\ell
\end{array}\right)=\frac{x(x-1) \cdots(x-\ell+1)}{\ell !}
$$

Proof. Putting

$$
F_{n}(x)=\sum_{\ell=0}^{n-1}(-1)^{\ell}\left(\begin{array}{c}
p+\ell \\
\ell
\end{array}\right)\left(\begin{array}{c}
p+n+x \\
n-\ell
\end{array}\right)
$$

and

$$
G_{n}(x)=\frac{x(x+1) \cdots(x+n-1)}{n !}
$$

$F_{n}(x)$ and $G_{n}(x)$ are polymomials of degree $n$.

We shall prove by induction on $n$; 


$$
F_{n}(x)=G_{n}(x) .
$$

If $n=1$, the assertion is obvious. For any polynomial $p(x)$, we define the difference operator $\Delta p$ by $\Delta p(x)=p(x)-p(x-1)$. Since $\Delta\left(\begin{array}{c}k+x \\ \ell\end{array}\right)=$ $\left(\begin{array}{c}k-1+x \\ \ell-1\end{array}\right)$, we have

$$
\Delta F_{n}(x)=F_{n-1}(x), \text { and } \Delta G_{n}(x)=G_{n-1}(x) .
$$

By the induction's hypothesis, $\Delta\left(F_{n}(x)-G_{n}(x)\right)=0$. Therefore $F(x)-G(x)$ must be a constant.

Since

$$
F_{n}(0)=\frac{1}{n !}\left(\sum_{\ell=0}^{n}(-1)^{\ell}\left(\begin{array}{l}
n \\
\ell
\end{array}\right)\right)(p+1)(p+2) \cdots(p+n)=0 \text { and } G_{n}(0)=0,
$$

the proof is thereby concluded.

The following lemma is an immediate consequence of Lemma 5.2.

LEMMA 5.3. Let $n$ and $m$ be positive integers and let $p$ be a non negative integer, then

$$
\begin{gathered}
\sum_{\ell=0}^{m} \frac{(-1)^{\ell}\left(\begin{array}{l}
n \\
\ell
\end{array}\right)}{(p+\ell+1)(p+\ell+2) \cdots(p+\ell+m)} \\
=\frac{m(m+1) \cdots(m+n-1)}{(p+1)(p+2) \cdots(p+n+m)} .
\end{gathered}
$$

Definition 5.2. Let $\mathfrak{V}=\left\{\left(U_{\alpha}, z_{\alpha}\right)\right\}$ be a projective coordinate covering of a compact Riemann surface $X$, let $\left\{q_{\alpha}\left(z_{\alpha}\right)\right\} \in \Gamma\left(\mathscr{Y}, \mathcal{O}\left(\kappa^{m}\right)\right)(m=2,3, \cdots, n)$ and let $\left\{\varphi_{\alpha}\left(z_{\alpha}\right)\right\}$ be vector valued holomorphic functions satisfying the relation (2.16) in each $U_{\alpha} \cap U_{\beta}$. We define the matrix valued holomorphic differential 1-forms $\left\{* \theta_{\alpha}^{[\varphi, q]}\left(z_{\alpha}\right)\right\}$ by

$$
\begin{aligned}
*_{\alpha}^{[\varphi, q]}\left(z_{\alpha}\right)= & \sum_{\ell=m}^{n}\left(\begin{array}{c}
n \\
\ell
\end{array}\right)\left(\begin{array}{c}
\ell \\
m
\end{array}\right) \frac{(\ell-1) !(2 m-1) !}{(m-1) !(m+\ell-1) !} \\
& \cdot\left[\left(\frac{d}{d z_{\alpha}}\right)^{\ell-m}\left(\left(\frac{d}{d z_{\alpha}}\right)^{n-\ell} \varphi_{\alpha}\left(z_{\alpha}\right) \cdot{ }^{t} \varphi_{\alpha}^{*}\left(z_{\alpha}\right)\right)\right] q_{\alpha}\left(z_{\alpha}\right) d z_{\alpha} .
\end{aligned}
$$

Moreover we introduce the matrix valued holomorphic functions $\left\{B_{m, \alpha}\left(z_{\alpha}\right)\right\}$ in each $U_{\alpha}$ as follows

$$
\begin{aligned}
B_{m, \alpha}\left(z_{\alpha}\right)= & \sum_{\ell=m+1}^{n} \sum_{k=1}^{\ell-m}(-1)^{k-1}\left(\begin{array}{l}
n \\
\ell
\end{array}\right)\left(\begin{array}{l}
\ell \\
k
\end{array}\right) \frac{(\ell-1) !(2 m-1) !}{(m-1) !(m+\ell-1) !} \\
& \cdot\left(\frac{d}{d z_{\alpha}}\right)^{\ell-m-k} q_{\alpha}\left(z_{\alpha}\right)\left(\frac{d}{d z_{\alpha}}\right)^{k-1}\left(\left(\frac{d}{d z_{\alpha}}\right)^{n-\ell} \varphi_{\alpha}\left(z_{\alpha}\right)^{t} \varphi_{\alpha}^{*}\left(z_{\alpha}\right)\right) .
\end{aligned}
$$


Proposition 5.1. In each intersection $U_{\alpha} \cap U_{\beta}$, the following relations hold.

(i ) $* \theta_{\alpha}^{[\varphi, q]}\left(z_{\alpha}\right)=\operatorname{Ad}\left(\rho_{\alpha \beta}\right) * \theta_{\beta}^{[\varphi, q]}\left(z_{\beta}\right)$

(ii) $\theta_{\alpha}^{[\varphi, q]}-* \theta_{\alpha}^{[\varphi, q]}=d B_{m, \alpha}$

Proof. First of all, we shall prove

$$
* \theta_{\alpha}^{[\varphi, q]}\left(z_{\alpha}\right)=(-1)^{n-m} \frac{(2 m-1) !((n-1) !)^{2}}{(n+m-1) !((m-1) !)^{2}}\left\langle\varphi_{\alpha}\left(z_{\alpha}\right), \varphi_{\alpha}^{*}\left(z_{\alpha}\right)\right\rangle^{n-m} d z_{\alpha} .
$$

By virtue of Lemma 5.3 it follows that

$$
\begin{aligned}
\sum_{\ell=0}^{n-m-k}(-1)^{\ell}\left(\begin{array}{c}
n-m-k \\
\ell
\end{array}\right) \frac{1}{(\ell+k+m)(\ell+k+m+1) \cdots(\ell+k+2 m-1)} \\
\\
\quad \frac{(n-k-1) !(k+m-1) !}{(m-1) !(n+m-1) !} .
\end{aligned}
$$

Hence we have;

$$
\begin{aligned}
* \theta_{\alpha}^{[\varphi, q]}\left(z_{\alpha}\right)= & \sum_{k=0}^{n-m} \frac{(2 m-1) ! n !}{(m-1) ! m !}(-1)^{k}(n-k-1) !(m+k-1) ! \\
& \cdot\left(\begin{array}{c}
n-m \\
k
\end{array}\right)\left(\frac{d}{d z_{\alpha}}\right)^{n-m-k} \varphi_{\alpha}\left(z_{\alpha}\right)^{t}\left(\left(-\frac{d}{d z_{\alpha}}\right)^{k} \varphi_{\alpha}^{*}\left(z_{\alpha}\right)\right) d z_{\alpha} .
\end{aligned}
$$

On the other hand, the $n-m$-th transvectant for vector valued functions $\varphi_{\alpha}\left(z_{\alpha}\right)$ and $\varphi_{\alpha}^{*}\left(z_{\alpha}\right)$ is given by

$$
\begin{aligned}
\left\langle\varphi_{\alpha}\left(z_{\alpha}\right), \varphi_{\alpha}^{*}\left(z_{\alpha}\right)\right\rangle^{n-m} & \\
= & \frac{1}{((n-1) !)^{2}} \sum_{k=0}^{n-m}(-1)^{k+n-m}(n-k-1) !(m+k-1) ! \\
& \cdot\left(\begin{array}{c}
n-m \\
k
\end{array}\right)\left(\frac{d}{d z_{\alpha}}\right)^{n-m-k} \varphi_{\alpha}\left(z_{\alpha}\right)^{t}\left(\left(\frac{d}{d z_{\alpha}}\right)^{k} \varphi_{\alpha}^{*}\left(z_{\alpha}\right)\right)
\end{aligned}
$$

comparing (5.16) and (5.17), we obtain (5.15).

By a property of the transvectant, we conclude

$$
* \theta_{\alpha}^{[\varphi, q]}\left(z_{\alpha}\right)=\operatorname{Ad}\left(\rho_{\alpha \beta}\right) * \theta_{\beta}^{[\varphi, q]}\left(z_{\beta}\right) .
$$

By a direct computation, one can verify the part (ii).

Q.E.D.

We denote by $\operatorname{Ad}_{0} \rho$ the flat vector bundle over $X$ defined by the transition functions $\operatorname{Ad}_{0} \rho_{\alpha \beta}$ acting on the space of $n \times n$ matrices of trace zero.

If we normarize the vector valued function $\varphi_{\alpha}\left(z_{\alpha}\right)$ so that the Wronskian 
matrix $F_{\alpha}\left(z_{\alpha}\right)$ of $\varphi_{\alpha}\left(z_{\alpha}\right)$ is equal to the unit matrix at a point $p$ in $U_{\alpha}$, from (5.17) (resp. (5.13)) it follows that

$$
T_{r}\left(* \theta_{\alpha}^{[\varphi, q]}\left(z_{\alpha}\right)\right)=0 \quad\left(\operatorname{resp} . T_{r}\left(\theta_{\alpha \alpha}^{[\varphi, q]}\left(z_{\alpha}\right)\right)=0\right) .
$$

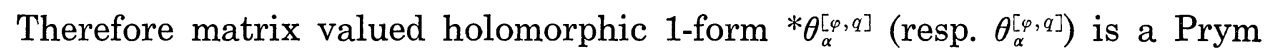
differential contained in the space $\Gamma\left(X, \mathcal{O}^{1,0}\left(\operatorname{Ad}_{0} \rho\right)\right)$.

Lemma 5.4. Let $\left\{\theta_{\alpha}^{\left[\varphi, \theta_{m j}\right]}\left(z_{\alpha}\right)\right\}$ be matrix valued holomorphic differential 1-forms in each coordinate open set $\left(U_{\alpha}, z_{\alpha}\right)$ by (5.13), and after passing to a refinement of the covering $\mathfrak{A}$ if necessary, select holomorphic matrix valued functions $G_{\alpha}\left(z_{\alpha}\right) \in \mathcal{O}_{U_{\alpha}}^{n \times n}$ such that

$$
-d G_{\alpha}\left(z_{\alpha}\right)=\theta_{\alpha}^{\left[\varphi, \theta_{m j}\right]}\left(z_{\alpha}\right) \quad \text { and } \quad G_{\alpha}\left(p_{\alpha}\right)=0
$$

where $p_{\alpha}$ is a point in $U_{\alpha}$.

Then the following variational formulas hold in each $U_{\alpha}$;

$$
\left.\frac{\partial}{\partial t_{m j}} \varphi_{\alpha}\left(z_{\alpha}, t\right)\right|_{t=0}=G_{\alpha}\left(z_{\alpha}\right) \varphi_{\alpha}\left(z_{\alpha}\right)
$$

Proof. Since the vector valued functions $\left.\left(\partial / \partial t_{m j}\right) \varphi_{\alpha}\left(z_{\alpha}, t\right)\right|_{t=0}$ satisfy the differential equation (5.11) in $U_{\alpha}$, an easy calculation shows

$$
\left.\frac{\partial}{\partial t_{m j}} \varphi_{\alpha}\left(z_{\alpha}, t\right)\right|_{t=0}=\left(-\int_{p_{\alpha}}^{z_{\alpha}} \theta_{\alpha}\left[\varphi, \theta_{m j}\right]\right) \cdot \varphi_{\alpha}\left(z_{\alpha}\right) \text {. }
$$

We shall consider the complex variety $V=N / S L(n, C)$;

$$
N=\left\{\left(X_{1}, \cdots, X_{g}, Y_{1}, \cdots, Y_{g}\right) \in S L(n, C)^{2 g} \mid\left[X_{1}, Y_{1}\right], \cdots,\left[X_{g}, Y_{g}\right]=1\right\}
$$

where [,] stands for the commutation and $S L(n, C)$ acts on $N$ by the inner automorphisms. By Theorem 2.2, any element of $\oplus_{m=2}^{n} \Gamma\left(X, \mathcal{O}\left(\kappa^{m}\right)\right)$ defines a flat vector bundle $\left\{\rho_{\alpha \beta}\right\}$ on $X$.

We define the complex analytic mapping

$$
\begin{aligned}
\theta: \bigoplus_{m=2}^{n} \Gamma\left(X, \mathcal{O}\left(\kappa^{m}\right)\right) & \longrightarrow V \\
\mathcal{U} & \\
\left(\theta_{2}, \cdots, \theta_{m}\right) & \longmapsto\left(\rho\left(\sigma_{1}\right), \cdots, \rho\left(\sigma_{g}\right), \rho\left(\tau_{1}\right), \cdots, \rho\left(\tau_{g}\right) \bmod S L(n, C)\right.
\end{aligned}
$$

where $\rho$ denotes the representation associated with the flat vector bundle defined by the transition functions $\left\{\rho_{\alpha \beta}\right\}$.

TheOREM 5.1. Let $\left\{U_{\alpha}, z_{\alpha}\right\}$ be a projective coordinate covering of a Riemann surface $X$, and let $\left\{\rho_{\alpha \beta}(t)\right\}$ be cocycles defined by (5.7), then the 
following variational formulas hold in each intersection $U_{\alpha} \cap U_{\beta}$;

$$
\begin{gathered}
\left.\rho_{\alpha \beta}(t)^{-1} \frac{\partial}{\partial t_{m j}} \rho_{\alpha \beta}(t)\right|_{t=0}=\operatorname{Ad}\left(\rho_{\alpha \beta}^{-1}\right) G_{\alpha}\left(z_{\alpha}\right)-G_{\beta}\left(z_{\beta}\right) \\
1 \leqq j \leqq \operatorname{dim} \Gamma\left(X, \mathcal{O}\left(\kappa^{m}\right)\right) .
\end{gathered}
$$

Proof. Since $\varphi_{\alpha}\left(z_{\alpha}, t\right)=\lambda_{\alpha \beta}^{n-1} \rho_{\alpha \beta}(t) \varphi_{\alpha}\left(z_{\beta}, t\right)$, we have

$$
\left.\frac{\partial}{\partial t_{m j}} \varphi_{\alpha}\left(z_{\alpha}, t\right)\right|_{t=0}=\left.\lambda_{\alpha \beta}^{n-1} \frac{\partial \rho_{\alpha \beta}}{\partial t_{m j}}(t)\right|_{t=0} \varphi_{\beta}\left(z_{\beta}\right)+\left.\lambda_{\alpha \beta}^{n-1} \rho_{\alpha \beta} \frac{\partial}{\partial t_{m j}} \varphi_{\beta}\left(z_{\beta}, t\right)\right|_{t=0} .
$$

By Lemma 5.2 it follows that

$$
\left.\lambda_{\beta \alpha}^{n-1} \rho_{\alpha \beta}^{-1} \frac{\partial}{\partial t_{m j}} \varphi_{\alpha}\left(z_{\alpha}, t\right)\right|_{t=0}=\rho_{\alpha \beta}^{-1} G_{\alpha}\left(z_{\alpha}\right) \rho_{\alpha \beta} \varphi_{\beta}\left(z_{\beta}\right)
$$

and

$$
\left.\frac{\partial}{\partial t_{m j}} \varphi_{\beta}\left(z_{\beta}, t\right)\right|_{t=0}=G_{\beta}\left(z_{\beta}\right) \varphi_{\beta}\left(z_{\beta}\right) .
$$

Hence we can conclude

$$
\left.\left.\rho_{\alpha \beta}(t)^{-1} \frac{\partial}{\partial t_{m j}} \rho_{\alpha \beta}\right|_{t=0} \varphi_{\beta}\left(z_{\beta}\right)=\left(\operatorname{Ad}\left(\rho_{\alpha \beta}^{-1}\right) G_{\alpha}\left(z_{\alpha}\right)-G_{\beta}\left(z_{\beta}\right)\right) \varphi_{\beta}\right)\left(z_{\beta}\right) .
$$

This completes the proof.

Q.E.D.

We shall investigate the differential equation (5.11) in a small neighborhood of the origin in the parameter space $t$ (see (5.6)).

Lemma 5.5. Let $X(z), Y(z)$ be vector valued functions defined as follows

$$
X(z)=\left[\begin{array}{c}
\vdots \\
z^{(n-1-a)} \\
\vdots
\end{array}\right], \quad Y(z)=\left[\begin{array}{c}
\vdots \\
(-1)^{a} z^{(a)} \\
\vdots
\end{array}\right]
$$

where $a=0,1,2, \cdots, n-1$ and $z^{(a)}=z^{a} / a !$.

We introduce the $n \times n$ matrix valued function $M_{n(m)}(z)$ by

$$
M_{n, m}(z)=\langle X(z), Y(z)\rangle^{n-m}
$$

where $\langle,\rangle^{n-m}$ stands for the $n-m$-th transvectant.

Then we obtain;

(1) the maximum degree of components in $M_{n, m}(z)$ is equal to $2(m-1)$

(2) $\operatorname{Tr}\left(M_{n, i}(z), M_{n, k}(w)\right)=c_{k} \delta_{i k}(z-w)^{2(k-1)}$ 
where $\delta_{i_{k}}$ is the Kronecker delta and $c_{k}$ is a non zero rational constant dependent sololy on $(i, n)$.

Proof. From definition the following properties hold

(i ) $X\left(\frac{a z+b}{c z+d}\right)=(c z+d)^{1-n} A_{\sigma} X(z)$

(ii) $\quad Y\left(\begin{array}{l}a z+b \\ c z+d\end{array}\right)=(c z+d)^{1-n t} A_{\sigma}^{-1} Y(z)$

where $\sigma=\left(\begin{array}{ll}a & b \\ c & d\end{array}\right) \in S L(2, C)$ and $\sigma \rightarrow A_{\sigma}$ is the $n$-th symmetric representation of $S L(2, C)$.

By virtue of Proposition 3.1. we have

$$
M_{n, m}\left(\frac{a z+b}{c z+d}\right)=(c z+d)^{-2(m-1)} \operatorname{Ad}\left(A_{\sigma}\right) M_{n, m}(z) .
$$

In particular, putting $\sigma_{1}=\left(\begin{array}{ll}1 & p \\ 0 & 1\end{array}\right)(p \in C), \sigma_{2}=\left(\begin{array}{rr}0 & 1 \\ -1 & 0\end{array}\right)$ we have

$$
M_{n, m}(z+p)=A_{\sigma_{1}} M_{n, m}(z) A_{\sigma_{1}}^{-1}
$$

and

$$
M_{n, m}(-1 / z)=z^{-2(m-1)} A_{\sigma_{2}} M(z) A_{\sigma_{2}}^{-1} .
$$

It follows from (5.20) that the maximum degree $d$ of components in the matrix $M_{n, m}(z)$ is not greater than $2(m-1)$. If $d<2(m-1)$, again by (5.20) we have $M_{n, m}(0)=0$. Since a straightforward computation shows that the $(n, n-m+1)$ component in the matrix $M_{n, m}(z)$ is not zero, the maximum degree of components in the matrix $M_{n, m}(z)$ is equal to $2(m-1)$. From (5.20), (5.21), for any non zero complex numbers $z$, $w$ we have

$$
\begin{aligned}
& \operatorname{Tr}\left(M_{n, i}(z), M_{n, k}(w)\right)=\operatorname{Tr}\left(M_{n, i}(z-w), M_{n, k}(0)\right) . \\
& \operatorname{Tr}\left(M_{n, i}(-1 / z), M_{n, k}(-1 / w)\right)=z^{-(m-1)} w^{-2(k-1)} \operatorname{Tr}\left(M_{n, i}(z), M_{n, k}(w)\right) \\
& \operatorname{Tr}\left(M_{n, i}\left(\frac{w-z}{z w}\right), M_{n, k}(0)\right)=\operatorname{Tr}\left(M_{n, i}(-1 / z), M_{n, k}(-1 / w)\right) .
\end{aligned}
$$

Hence we conclude;

$$
\operatorname{Tr}\left(M_{n, i}(z), M_{n, k}(w)\right)=c_{k} \delta_{i k}(z-w)^{2(k-1)}
$$

where $c_{k}$ is a constant.

We shall show that the constant $c_{k}$ is not zero.

By a direct computation the $(p, q)$ component $M_{n, m}(z)$ in the matrix $M_{n, m}(z)$ is given as follows, 


$$
\begin{aligned}
M_{n, m}(z)_{p, q}= & \sum_{\ell=m}^{n}(-1)^{\ell-m}\left(\begin{array}{l}
n \\
\ell
\end{array}\right)\left(\begin{array}{l}
\ell \\
m
\end{array}\right) \\
& \cdot \frac{(\ell-1) !(2 m-1) !}{(m-1) !(m+\ell-1) !}\left(\frac{\ell+q-p-1}{q-1}\right) z^{(m+q-p-1)}
\end{aligned}
$$

where we denote for any integer $\ell$

$$
z^{(\ell)}= \begin{cases}\frac{z^{\ell}}{\ell !} & \ell>0 \\ 0 & \ell \leqq 0\end{cases}
$$

Since the degree of the $(p, q)$ component in the matrix $M_{n, m}(z)$ is $m-1$ if and only if $p=q$, the coefficient of $2^{m-1} w^{m-1}$ in the polynomial $\operatorname{Tr}\left(M_{n, m}(z)\right.$, $M_{n, m}(w)$ ) of 2 variables $z, w$ is equal to

$$
\sum_{p=1}^{n}\left(\sum_{\ell=m}^{n}(-1)^{\ell-m}\left(\begin{array}{l}
n \\
\ell
\end{array}\right)\left(\begin{array}{l}
\ell \\
m
\end{array}\right) \frac{(\ell-1) !(2 m-1)}{(m-1) !(m+\ell-1) !}\left(\begin{array}{l}
\ell-1 \\
p-1
\end{array}\right)\right)^{2} .
$$

Since

$$
\begin{aligned}
\sum_{\ell=m}^{n} & (-1)^{\ell-m}\left(\begin{array}{c}
n \\
\ell
\end{array}\right)\left(\begin{array}{c}
\ell \\
m
\end{array}\right) \frac{(\ell-1) !(2 m-1) !}{(m-1) !(m+\ell-1) !} \\
& =\left(\begin{array}{l}
n \\
m
\end{array}\right) \frac{(2 m-1) !}{(m-1) !} \sum_{\ell=0}^{n-m}(-1)^{\ell}\left(\begin{array}{c}
n-m \\
\ell
\end{array}\right) \frac{1}{(\ell+m)(\ell+m+1) \cdots(\ell+2 m-1)} \\
& =\left(\begin{array}{l}
n \\
m
\end{array}\right) \frac{(2 m-1) !}{(m-1) !} \frac{(n-1) !}{(n+m-1) !}
\end{aligned}
$$

the coefficient of $z^{m-1} w^{m-1}$ in the polynomial $\operatorname{Tr}\left(M_{n, m}(z), M_{n, m}(w)\right)$ is not zero. This means that the constant $c_{k}$ is not zero.

Q.E.D.

Let us introduce vector valued holomorphic functions $X_{\alpha}\left(z_{\alpha}\right), Y_{\alpha}\left(z_{\alpha}\right)$, $M_{n, m, \alpha}\left(z_{\alpha}\right)$ in each projective coordinate open set $\left(U_{\alpha}, z_{\alpha}\right)$ by

$$
X_{\alpha}\left(z_{\alpha}\right)=X\left(z_{\alpha}\right), \quad Y_{\alpha}\left(z_{\alpha}\right)=Y\left(z_{\alpha}\right) \quad \text { and } \quad M_{n, m, \alpha}\left(z_{\alpha}\right)=M_{n, m}\left(z_{\alpha}\right) .
$$

Then $\left\{X_{\alpha}\left(z_{\alpha}\right)\right\}$ correspond to semi-canonical form

$$
L_{n, \alpha}\left(p_{\alpha} \mid z_{\alpha}\right)=\left(\frac{d}{d z_{\alpha}}\right)^{n}
$$

and $Y_{\alpha}\left(z_{\alpha}\right)=X_{\alpha}^{*}\left(z_{\alpha}\right)$.

By virtue of Proposition 5.1 for any element $\theta_{m, \alpha}\left(z_{\alpha}\right) \in \Gamma\left(\mathscr{U}, \mathcal{O}\left(\kappa^{m}\right)\right)$,

$$
\left(M_{n, m, \alpha}\left(z_{\alpha}\right) \theta_{m, \alpha}\left(z_{\alpha}\right)\right) \in \Gamma\left(\mathfrak{H}, \operatorname{Ad}_{0} \rho\right)
$$

and 


$$
\operatorname{Tr}\left(M_{n, m, \alpha}\left(z_{\alpha}\right), M_{n, \ell, \alpha}\left(w_{\alpha}\right)\right)=c_{m} \delta_{m, \ell}\left(z_{\alpha}-w_{\alpha}\right)^{2(m-1)} .
$$

Definition 5.3. The $C$-linear mapping $\beta_{m}$ from the complex vector space of holomorphic $m$-differentials $\Gamma\left(X, \mathcal{O}\left(\kappa^{m}\right)\right)$ to the vector space $\Gamma\left(X, \mathcal{O}^{1,0}\left(\operatorname{Ad}_{0} \rho\right)\right) / d \Gamma\left(X, \mathcal{O}\left(\operatorname{Ad}_{0} \rho\right)\right)$ is defined by

$$
\begin{aligned}
\beta_{m}: & \Gamma\left(X, \mathcal{O}\left(\kappa^{m}\right)\right) \longrightarrow \Gamma\left(X, \mathcal{O}^{1,0}\left(\operatorname{Ad}_{0} \rho\right)\right) / d \Gamma\left(X, \mathcal{O}\left(\operatorname{Ad}_{0} \rho\right)\right) \\
& \left\{\theta_{m, \alpha}\left(z_{\alpha}\right)\right\} \longmapsto\left\{M_{n, m, \alpha}\left(z_{\alpha}\right) \theta_{m, \alpha}\left(z_{\alpha}\right)\right\} / \bmod d \Gamma\left(X, \mathcal{O}\left(\operatorname{Ad}_{0} \rho\right)\right) .
\end{aligned}
$$

TheOREm 5.2. Let $\theta$ be the complex analytic mapping defined by (5.19);

$$
\theta: \bigoplus_{m=2}^{n} \Gamma\left(X, \mathcal{O}\left(\kappa^{m}\right)\right) \longrightarrow V
$$

and let $\rho$ be the $n$-th symmetric tensor representation of the fundamental group $\pi_{1}(X)$. Then the mapping $\theta$ is non-singular at the origin $(0, \cdots, 0)$ $\in \oplus \Gamma\left(X, \mathcal{O}\left(\kappa^{m}\right)\right)$ and the tangent space to the image at the point $\rho \in V$ can be identified with the $\left(n^{2}-1\right)(g-1)$-dimensional subspace of $H^{1}\left(X, \operatorname{Ad}_{0} \rho\right)$ consisting of the period classes of the Prym differentials $\Gamma\left(X, \mathcal{O}^{1,0}\left(\operatorname{Ad}_{0} \rho\right)\right)$.

Proof. By virtue of Lemma 5.1 and Theorem 5.1, the tangent vector

$$
\left.\rho_{a \beta}(t)^{-1} \frac{\partial}{\partial t_{m j}} \rho_{\alpha \beta}(t)\right|_{t=0} \in H^{1}\left(X,\left(\operatorname{Ad}_{0} \rho\right)\right)
$$

is equal to the period class of the Prym differential $\left\{M_{n, m}\left(z_{\alpha}\right) \theta_{m, \alpha}\left(z_{\alpha}\right)\right\}$ up to a non-zero constant. By (5.23) and recalling $\left\{\theta_{m j, \alpha}\right\}\left(1 \leqq j \leqq \operatorname{dim} \Gamma\left(X, \mathcal{O}\left(\kappa^{m}\right)\right)\right.$ is a basis of $\Gamma\left(X, \mathcal{O}\left(\kappa^{m}\right)\right)$, we can conclude that the image in $H^{1}\left(X, \operatorname{Ad}_{0} \rho\right)$ of the mapping on the tangent space induced by the mapping $\theta: \oplus \Gamma\left(X, \mathcal{O}\left(\kappa^{m}\right)\right)$ $\rightarrow V$ is the space of period classes of the Prym differentials $\Gamma\left(X, \mathcal{O}^{1,0}\left(\operatorname{Ad}_{0} \rho\right)\right)$. Since it follows from the Riemann-Roch theorem that

$$
\operatorname{dim} \bigoplus_{m=2}^{n} \Gamma\left(X, \mathcal{O}\left(\kappa^{m}\right)\right)=\left(n^{2}-1\right)(g-1)
$$

and

$$
\operatorname{dim} \Gamma\left(X, \mathcal{O}^{1,0}\left(\operatorname{Ad}_{0} \rho\right)\right) / d \Gamma\left(X, \mathcal{O}\left(\operatorname{Ad}_{0} \rho\right)\right)=\left(n^{2}-1\right)(g-1),
$$

the mapping $\theta$ is non-singular. This completes the proof.

Q.E.D.

By Theorem 5.2, the mapping

$$
\oplus \beta_{m}: \oplus \Gamma\left(X, \mathcal{O}\left(\kappa^{m}\right)\right) \longrightarrow \frac{\Gamma\left(X, \mathcal{O}^{1,0}\left(\operatorname{Ad}_{0} \rho\right)\right)}{d \Gamma\left(X, \mathcal{O}\left(\operatorname{Ad}_{0} \rho\right)\right.}
$$

is an isomorphism. Hence the following diagram holds: 


$$
\begin{aligned}
& 0 \longrightarrow \oplus_{m=2}^{n} \Gamma\left(X, \mathcal{O}\left(\kappa^{m}\right)\right) \longrightarrow \oplus H^{1}\left(X, P_{2(m-1)}\left(\kappa^{-(m-1)}\right)\right) \longrightarrow \oplus \Gamma\left(X, \mathcal{O}\left(\kappa^{m}\right)\right)^{*} \longrightarrow 0 \\
& \text { । } \oplus \beta_{m} \quad \text { । } \oplus \beta_{m}^{*} \\
& 0 \longrightarrow \frac{\Gamma\left(X, \mathcal{O}^{1,0}\left(\operatorname{Ad}_{0} \rho\right)\right.}{d \Gamma\left(X, \mathcal{O}\left(\operatorname{Ad}_{0} \rho\right)\right)} \longrightarrow H^{1}\left(X, \operatorname{Ad}_{0} \rho\right) \longrightarrow\left[\frac{\Gamma\left(X, \mathcal{O}^{1,0}\left(\operatorname{Ad}_{0} \rho\right)\right)}{d \Gamma\left(X, \mathcal{O}\left(\operatorname{Ad}_{0} \rho\right)\right.}\right]^{*} \longrightarrow 0
\end{aligned}
$$

where lows sequences are exact and [ ]* stands for the dual vector space. Hence two vector spaces $\oplus H^{1}\left(X, P_{2(m-1)}\left(\kappa^{-1}\right)\right)$ and $H^{1}\left(X, \operatorname{Ad}_{0} \rho\right)$ are canonically isomorphic. (See [4], [6])

\section{REFERENCES}

[1] H. Morikawa, Some analytic and geometric applications of the invariant theoretic method, Nagoya Math. J., 80 (1980), 1-47.

[2] D. A. Hejhal, The variational theory of linearly polynomic functions, J. Analyse Math., 30 (1976), 215-264.

[ 3 ] —_, Monodromy groups and Poincaré series, Bull. Amer. Math. Soc., 84 (1978), $339-376$.

[ 4 ] R. C. Gunning, Lectures on Riemann Surfaces, Princeton Univ. Press, (Mathematical Notes 2), 1966.

[5] — Analytic structures on the space of flat vector bundles over a compact Riemann surface, Several Complex Variables II, Maryland, 1970. Springer Lecture Notes 185 (1971), 47-62.

[6] _ L Lectures on Vector Bundles over Riemann Surfaces, Princeton University Press, 1967.

Department of Mathematics

Faculty of Science

Nagoya University

Chikusa-ku, Nagoya 464

Japan 